\title{
Über die Algenwelt der Höhlen in Ungarn
}

\author{
Von P. PALIK ${ }^{1}$ )
}

Mit Tafel 9 (1)

Das Studium der Algen der dunklen Höhlen haben wir mit G. Claus und E. Suba im Juli 1952 in der Baradla-Höhle begonnen.

Diese Höhle befindet sich im Norden des Landes, im Karstgebiet von Gömör-Torna. Das ganze Gebiet ist eben. Aus ihm erhebt sich der sogenannte Poronyatetö zu einer Höhe von $500 \mathrm{~m}$. Die Länge der heute bereits bekannten Gänge des Höhlensystems beträgt $22 \mathrm{~km}$; von diesen entfällt ein Abschnitt von $15 \mathrm{~km}$ auf ungarisches Gebiet, die $7 \mathrm{~km}$ lange Domica befindet sich aber in der Tschechoslowakei. Der Eingang der Höhle bei Aggtelek liegt in $332 \mathrm{~m}$ Seehöhe. Die Baradla ist eine ,Durchgangshöhle“. Zwei unterirdische Bäche, der Styx und der Acheron, fließen durch sie. Diese vereinigen sich, ihr Wasser stürzt dann in eine untere Höhle und kommt als die Quelle von Jósvafö an die Erdoberfläche. Der pH-Wert des Wassers der Höhle ist 7,4. Ihre Temperatur ist gleich der der Höhle (im Durchschnitt $10^{\circ} \mathrm{C}$ ). Die relative Luftfeuchtigkeit in der Höhle liegt bei 84-100\% (Dudich 4; S. 130). Abgesehen von der Umgebung des Einganges, ist die Höhle völlig dunkel. Auf unser Ersuchen hat der Assistent Zoltan TárczyHornoch auch Strahlungsmessungen in der Baradla vorgenommen. Die Messungen ergaben keine stärkere Radioaktivität in der Höhle. An einigen Stellen ist die Strahlung auffallend gering, nirgends bedeutender als der Durchschnitt in Budapest. Der basische Kalkstein, die Stalaktite und Stalagmite haben 0-Aktivität, die Sedimente, besonders der Lehm, zeigen geringe Gammaaktivität. Betastrahlung konnte im ganzen Gebiet nicht nachgewiesen werden (Claus 1).

$\mathrm{Zu}$ unseren Algenuntersuchungen haben wir Material teils von den Felsen, von den Tropfsteinen, von der Wand der Höhle, von deren Decke, von den sogenannten ,,Rußflecken“, ferner von den ,,Kerzen“ abgekratzt. Wir haben aber auch aus den Bächen, aus mit Wasser gefüllten Gruben, selbst aus dem „Aquarium“ gesammelt und dieses Material immer mit völlig neuen Planktonnetzen durchgesiebt. Wir

1) Mikrobiologisches Institut der Eötvös Loránd Universität, Budapest. 
haben das Material in sterilen Glasdosen, in schwarzes Papier eingepackt, transportiert.

Die Untersuchung des hier gesammelten Materials wurde von G. Claus durchgeführt. In seinem Bericht (1) erwähnt er 69 Algenarten, Varietäten und Formen, von denen 44 Arten, Varietäten und Formen, das heißt 64\% des eingesammelten Materials, zum Phylum der Cyanophyten (Blaualgen) gehören. 1 Art gehört dem Stamm der Euglenophyten an, 12 Arten und Varietäten sind Chrysophyten, 12 Arten gehören zu den Chlorophyten.

Wir haben die Baradla später noch mehrmals besucht, um Algen zu sammeln. Bei einer Gelegenheit (1959) haben wir an einer Stelle, die „Láva-folyó “2) genannt wird, im abgekratzten Material von G. Kertész eine interessante Blaualgenart gefunden. Diese Art hat sich im Verlaufe der weiteren Untersuchungen als eine bisher unbekannte neue Art erwiesen. Nach den Untersuchungen erhielt diese Alge den Namen Baradlaia speluncaecola Palik (19). Wir fanden diese Alge in vegetativer Form vor, einzelne vermehrten sich sogar, sie erschien also nicht als Dauerzelle. Diese Art war in Gesellschaft von 3 Blaualgenarten (Gloeocapsa punctata Näg., Gloeocapsa mellea Kütz., Synechococcus elongatus Näg.). Diese 3 Arten werden auch von Claus (1) erwähnt. Die vorerwähnte Art besteht aus dünnen, 1,2-1,5 $\mu$ breiten, biegsamen, blaugrünen, meistens verzweigten Fäden, die, stellenweise miteinander verflochten, Lager bilden (Fig. 4a). Später lagert sich in den Scheiden der Fäden Kalk ab, was an einzelnen Exemplaren so beträchtlich sein kann, daß die Fäden auch die Dicke von $5 \mu$ erreichen können (Fig.4e). Infolge der stellenweise beträchtlicheren Kalkablagerungen nehmen die Fäden manchmal Knüppel- oder Kreuzform an (Fig. 4e, 4f). Die Länge der Fäden wechselte zwischen 9-80 2 . Da diese Blaualgenfäden in bedeutender Menge Kalk ausscheiden, spielen sie in der Gestaltung des Kalktuffs eine wichtige Rolle.

$\mathrm{Zu}$ Zwecken algologischer Untersuchungen haben wir 1953 die Höhle von Abaliget besucht. Auch in dieser haben wir mehrfach Material gesammelt.

Die Höhle von Abaliget befindet sich im Komitat Baranya, am nordwestlichen Rand des Mecsek-Gebirges. Ihr Eingang liegt am Fuße des Bodó-Berges, 209,5 m über dem Meeresspiegel. Wir kennen die Höhle aus der Beschreibung von Gebhardt (10). Die vorherrschenden Gesteine der Höhle bestehen im allgemeinen aus Kalkstein. Die Tropfsteinbildung ist hier unbedeutend, aber Spuren davon sind längs

$\left.{ }^{2}\right)$ „Láva-folyó“ (Lavafluß) ist eine Kalktuff-Formation $1200 \mathrm{~m}$ vom Aggteleker Eingang der Baradla-Höhle entfernt. 
der ganzen Höhle anzutreffen. Nach der Einteilung von Venkovits handelt es sich hier um eine sogenannte „Quellenhöhle“. Der Eingang liegt nach Norden, ist etwa $2 \mathrm{~m}$ hoch, aber ein ziemlich wasserreicher Bach, der aus ihm herausfließt, macht das Eindringen schwer. Der Hauptgang der Höhle ist 466,8 $\mathrm{m}$ lang, im Durchschnitt $3 \mathrm{~m}$ hoch und $2 \mathrm{~m}$ breit. An den Hauptgang schließen sich zwei Seitengänge an, der eine $40 \mathrm{~m}$ und der andere $60 \mathrm{~m}$ lang. Am Ende der Höhle finden wir einen See. Die Jahresmitteltemperatur ihrer Luft ist $12,6^{\circ} \mathrm{C}$. Die relative Luftfeuchtigkeit liegt bei $94 \%$ im Durchschnitt. Abgesehen von der Nachbarschaft des Einganges, herrscht in der Höhle infolge der scharfen Krümmungen völlige Dunkelheit. Der pH-Wert des Wassers des Baches ist 6,05.

Nach der Mitteilung von Dr. A. Urban wurden auch in dieser Höhle nach dem Jahre 1953 radiometrische Untersuchungen vorgenommen und niedrigere Werte als die der örtlichen Hintergrundstrahlung wahrgenommen. (Mit einem UR4M-Gamma-Instrument.) Die Radonkonzentration der Höhlenluft war 0,49-1,02 Eman, während der Urangehalt des Wassers 0,5-2,0 Mikrogramm/Liter, der Radiumgehalt 0,7.10-12 Curie/Liter und die Radiumemanation 0,15-0,47 Eman zur Zeit ihrer Untersuchungen ausmachten.

Im Bett des Höhlenbaches, aber auch über die Ufer erstreckt, in der Nähe des Sees, also in völliger Dunkelheit haben wir ein ChantransiaLager von 2-3 dm im Durchmesser angetroffen. Dieses Genus gehört zum Phylum der Rotalgen. Wir haben aber auch ausgedehnte Blaualgenlager, die aus Oscillatoria- und Symploca-Arten bestanden, an den Felsenwänden gesehen. Das Untersuchungsmaterial wurde auch hier nach der oben beschriebenen Weise gesammelt. Die Zahl der eingesammelten Arten war 93; es befanden sich unter ihnen 52 Arten von Cyanophyten (51\%), 2 Arten von Euglenophyten (ausgewachsene vegetative Zellen, nicht Cysten), 18 Arten von Chrysophyten, 1 Art von Pyrrophyten, 19 Arten von Chlorophyten, und 1 Art von Rhodophyten (Claus 5).

Aus früheren Untersuchungen ist es schon bekannt, daß die staubund allergenfreie Höhlenluft auf Asthmatiker und an Katarrh der Luftwege Erkrankte von wohltätiger Wirkung ist. In der jüngsten Zeit hat man auch in der Höhle von Abaliget Untersuchungen zum Studium der verschiedenen Heilwirkungen der Höhlen durchgeführt, und es sind in der Höhle in Verbindung damit bedeutende Änderungen vorgenommen worden. Man hat zum Beispiel die ganze Höhle mit elektrischer Beleuchtung versehen. Infolge dieser Tatsache sind in der 
Algenwelt der Höhle ohne Zweifel heute bereits bedeutende Wandlungen eingetreten.

Im Jahre 1954 begannen wir auch mit der Untersuchung der Algenflora der sogenannten Béke-Höhle (Friedenshöhle). Diese Höhle wurde von Jakucs und seinen Mitarbeitern im Jahre 1952 entdeckt (12). Wir waren die ersten Algologen, die einen großen Teil der bis dahin bekannten Abschnitte der Höhle begingen und darin Algenmaterial sammelten. Die Arbeit wurde hier in derselben Weise wie in der Baradla durchgeführt.

Die Béke-Höhle liegt in unmittelbarer Nachbarschaft der Baradla, etwa $4 \mathrm{~km}$ von deren Aggteleker Eingang entfernt. Sie ist wie die Baradla eine Karsterscheinung des Gömörer Karstgebietes. Nach der Feststellung von Jakucs ist sie eine, ,Durchgangshöhle“. Auch durch sie fließt ein Bach. Die erschlossene, gemessene und abgeschätzte Länge des Höhlensystems war 1953 nach der Arbeit von Jakucs 9,75 km. Die Luft ist fast gesättigt mit Dunst (98\%), ihre Temperatur schwankt zwischen 9,5 und $11^{\circ} \mathrm{C}$. Die Höhle ist ganz dunkel.

Es sind hier 90 Algenarten gefunden worden, von diesen sind 68 Cyanophyten (75\%), 2 Euglenophyten, 8 Chrysophyten, 11 Chlorophyten und 1 Art, die zum Phylum der Rhodophyten gehört.

Der Forscher J.Pónyi fand bei Gelegenheit seiner zoologischen Sammlungen einen schleimigen, hautartigen Überzug an den Steinen. Nach näherer Untersuchung stellte es sich heraus, daß der Überzug ein Lager einer Chantransia-Art war. 2 von den 90 eingesammelten Algenarten sind heute in arktischen und antarktischen Gebieten heimisch. Die in der Béke-Höhle gefundenen Algenarten zeigen nach der Feststellung von Claus nicht viel Übereinstimmung mit den Arten, die in der Baradla gesammelt wurden. Freilich gibt es auch gemeinsame Arten. Die Algenflora der Béke-Höhle ist viel reicher, sie erinnert an das Material, das in der Höhle von Abaliget gesammelt wurde. Auf Grund der Algenwelt können wir den Schluß ziehen, daß die Höhle von Abaliget und die Béke-Höhle wahrscheinlich zu derselben Zeit entstanden sind oder daß die Algenflora der beiden Gebiete zur Zeit der Entstehung der Höhlen vermutlich die gleiche war. Die Aggteleker Höhle scheint älter zu sein als die beiden anderen.

Auf unser Ersuchen hat Z. Tárczy-Hornoch auch in der BékeHöhle Strahlungsmessungen vorgenommen. Seine diesbezüglichen Festellungen stimmen mit den Messungen in der Baradla überein.

Die Untersuchung der Algenflora der Höhle von Pálvölgy wurde im April 1955 von É. Suba begonnen. In ihrer Arbeit schreibt sie folgendes (22): ,Diese Höhle liegt in Budapest, nordwestlich vom Rózsadomb (Rosenhügel). Ihre Länge beträgt etwa $1 \mathrm{~km}$. Nach der 
Feststellung von Venkovits und Jaskó gehört sie zu den sogenannten Zerklüftungshöhlen. Diese Höhle ist nämlich zur Zeit der tertiären Gebirgsbildung durch Spaltung entstanden. Die Spalte wurde dann durch Karstwasser und Heißquellen erweitert." Das Einsammeln des Untersuchungsmaterials erfolgte hier auch auf die bereits mehrmals erwähnte Weise. Die Zahl der eingesammelten Algenarten ist 41. Von diesen gehören 21 Arten dem Phylum Cyanophyta an (51\%), 7 Arten sind Chrysophyten, 13 Arten sind Chlorophyten; 8 Arten der Blaualgen bevorzugen wärmere Wasser. É. Suba meint, diese Arten seien wahrscheinlich Relikte aus der Zeit, als es noch Thermalquellen in der Höhle gab. 2 Arten der Grünalgen sind heute in kälteren Gegenden heimisch.

Im Jahre 1955 haben wir auch Algen aus der Höhle „,Kölyuk" in Mánfa untersucht. Diese Höhle befindet sich im Süden Ungarns, im Mecsek-Gebirge bei Pécs. Sie ist eine Karsthöhle. G. Magyari, der die Erschließung der Höhle leitet, teilte uns mündlich mit, daß der bis dahin bekannte Hauptabschnitt der Höhle ungefähr $200 \mathrm{~m}$ lang sei. Am Ende befinde sich ein kleiner See. Die Temperatur des Wassers liege bei $9,5-10^{\circ} \mathrm{C}$, die relative Luftfeuchtigkeit bei $71-99 \%$. Am kleinen See sei die Dunkelheit bereits vollkommen. Das nischenartige Ende der Höhle sei durch einen Siphon vom übrigen Teil der Höhle abgesperrt. Diese Nische könne man nur durch Schwimmen unter dem Siphon erreichen. Magyari hat Untersuchungsmaterial von der hinteren Wand dieser Nische abgekratzt. In diesem wurde eine neue Gomontiella-Art gefunden (eine Blaualge), die zu Ehren Magyaris den Namen Gomontiella magyariana (s. Claus 3) erhielt. Diese neue Gomontiella lebte in Gesellschaft von 19 anderen Blaualgen (71\%), 2 Kieselalgen- und 7 Grünalgenarten.

Im Zusammenhang mit der intensiven Forschungsarbeit werden immer wieder neue Höhlen in Ungarn entdeckt. Unter anderem befaßt man sich zur Zeit mit der Untersuchung der Algenflora der ,Vas-ImreHöhle", die ebenfalls im Bükk-Gebirge, in der Nähe der Baradle-Höhle liegt.

Die Botaniker und Pflanzenphysiologen haben die Untersuchung der Algenflora der dunklen Höhlen sowohl in der Vergangenheit als auch in der Gegenwart ziem]ich vernachlässigt. Dies ist natürlich, denn es schien fast unvorstellbar, daß in der ständigen Finsternis der Höhlen chlorophyllhaltige Pflanzen leben sollten.

Bei den in den dunklen Höhlen lebenden Algen erhebt sich die Frage, was für Energiequellen diese zur Abwicklung ihrer Lebensvorgänge verwenden. In diesem Zusammenhang haben sich bereits mehrere Hypothesen gebildet. 
1. Die erste wäre, daß sie die Energie der verschiedenen Strahlungen verwenden. Bei unseren Forschungen hatten wir dies auch angenommen, deshalb haben wir in der Baradla- und in der Béke-Höhle Strahlungsmessungen vorgenommen. Ähnliche Beobachtungen wurden auch in der Höhle von Abaliget durchgeführt. Die Messungen ergaben aber, daß die von den Pflanzen eventuell verwendbare Gammastrahlung sehr unbedeutend ist. Betastrahlung hingegen wurde gar nicht beobachtet. Diese können also bei der Assimilation keine Rolle spielen. Im Zusammenhang mit den Strahlungen können wir eventuell der von Prof. D. Fehér angenommenen Theorie der Mikrostrahlung beistimmen (8). Er gründete seine Vermutung darauf, daß seine Algenkulturen, die er auf längere Zeit (z. B. 1-2 Jahre) in den Boden versenkt, also in völliger Dunkelheit gehalten hatte, am Leben blieben, ihre Farbe nicht verloren, sich sogar fortpflanzten. Er meint, daß es eine Strahlung geben müsse, die wir mit unseren Instrumenten noch nicht messen könnten, die die assimilierende Tätigkeit der in der Dunkelheit gehaltenen Algenzellen möglich mache.

2. Auf Grund der Untersuchungen in den Höhlen der Schweiz vermutet Magdeburg (16) eine Symbiose zwischen den Algenzellen und den Eisenbakterien. Er meint, die Eisenbakterien versähen die Algenzellen mit Nährstoffen. In Anbetracht der Vermutung Magdeburgs haben wir bei unseren Forschungen den Eisenbakterien volle Aufmerksamkeit geschenkt. Diese erschienen aber in den einzelnen Höhlen in so minimaler Menge, daß sie hinsichtlich der Symbiose keine bedeutenden Faktoren darstellen können.

3. Es ist möglich, daß in völliger Dunkelheit lebende Algenarten solche organische Stoffe zur Nahrung verwenden, die bereits von einzelnen Bakterien und niederen Pilzen halb abgebaut worden sind. Experimente im Laboratorium haben nämlich erwiesen, daß einzelne Scenedesmus-Arten (vom Phylum der Grünalgen), in völliger Dunkelheit gehalten, Jahre hindurch am Leben bleiben. wenn sie halb abgebaute organische Stoffe in gelöstem Zustand reichlich zur Verfügung haben.

4. Die im Dunkeln lebenden Algen dürften ihre Lebensprozesse auch durch Chemosynthese abwickeln. Die Richtigkeit dieser Hypothese wurde von einigen Forschern (Gafron 9; Kessler 13) auch durch Experimente bestätigt. Im Verlauf ihrer Versuche hat sich erwiesen, daß solche Algen, die Hydrogenase enthalten, die unter dem Einfluß dieses Enzyms freiwerdende Energie verwerten. Nach unserer Meinung ist es möglich, daß auch die Chemosynthese bei einem Teil der in dunklen Höhlen lebenden Algen als Energiequelle eine Bedeutung hat, aber es 
ist nicht wahrscheinlich, daß die auf diese Weise freigewordene Energie genügte, um zum Beispiel Chantransia-Lager mit Durchmessern von 2-3 dm zu erhalten.

Das Problem des Lebens verschiedener Algenarten ohne Licht wurde schon vielfach untersucht. Man hat sich aber speziell mit Höhlenalgen bisher nicht befaßt. Man hat verschiedene Grünalgenarten untersucht (Scenedesmus, Ankistrodesmus, Chlorella), auch grüne Flagellaten (Euglena-Arten), die Blaualgen hat man aber nicht besonders studiert. Eine bedeutende Anzahl der im Dunkel der Höhlen heimischen Algenarten gehört aber, wie wir gesehen haben, zum Phylum der Cyanophyten. Es wäre also sehr erwünscht, auch mit Blaualgen Versuche anzustellen.

Die bisherigen Untersuchungen sind leider in Laboratorien durchgeführt worden. Es wäre wichtig, die Forschungen in den Höhlen selbst vorzunehmen, da wir dort am leichtesten gewährleisten können, daß wir die Kulturen unter den gleichen Umständen erhalten, wie die Arten auch in den Höhlen leben.

Bezüglich der höhlenbewohnenden Algenarten kann sich auch eine andere Frage erheben: Wie sind die aufgezählten Algenarten an ihren Standort gekommen?

Wir hegen die Vermutung, daß ein großer Teil von ihnen bereits gleichzeitig mit der Entstehung der Höhle hingekommen ist. Mehrere Tatsachen schienen diese Vermutung im Verlauf unserer Untersuchungen zu bestätigen.

\section{ZUSAMMENFASSUNG}

Das Vorstehende ist ein Bericht über die Erforschung der Algen, die in den Höhlen von Ungarn leben. Es wurden die Ergebnisse der Forschungen bezüglich der Algenflora von Baradla-, Bèke-, Abaliget-, Pálvölgy-, KölyukHöhle aufgezählt. Theorien über die möglichen Energiequellen, sowie Strahlung, Symbiosis, Chemosynthesis oder Auxotrophie, welche von diesen Algen, die in vollkommener Finsternis leben, verwendet werden, wurden erörtert. Die Frage der Ansiedlung der Algen in diesen Höhlen wurde besprochen.

\section{ABSTRACT}

An account of the researches carried out on the algae living in the caves of Hungary is given. The results of the investigations concerning the algal flora of the Baradla, Peace, Abaliget, Pálvölgy, Kölyuk caves are enumerated. Theories about the possible energy source utilized by these algae living in the complete darkness of caves such as radiation, symbiosis, chemosynthesis or auxothrophy are discussed. The question of the settling of algae into the caves is debated. 


\section{LITERATUR}

1. Claus, G. - 1955: Algae and Their Mode of Life in the Baradla Cave at Aggtelek. Acta. Bot. Acad. Sci. Hung. 2 (1-2): 1-27.

2. - 1959: Concerning the Algal Flora ot the Peace Cave at Aggtelek in Hungary (Abstract.) Proc. IX. Internat. Bot. Congr. 2: 75.

3. -- 1960: Re-evaluation of the Genus Gomontiella. Revue Algologique. Nouv. Ser. 5: 103-111.

4. - 1962 a: Data on the Ecology of the Algae of Peace Cave in Hungary. Nova Hedwigia 4 (1-2): 55-79.

5. - $1962 \mathrm{~b}$ : Beiträge zur Kenntnis der Algenflora der Abaligeter Höhle. Hydrobiologia 19 (2) : 192-222.

6. Dudich, E. - 1932 : Biologie der Aggteleker Tropfsteinhöhle Baradla in Ungarn, pp. 1-246. Wien.

7. - 1960: Das höhlenbiologische Laboratorium der Eötvös-Loránd-Universität. Ann. Univ. Sci. Budapestiensis Rol. Eötvös nom. Sect. Biol. 3: 131-135. Budapest.

8. FenÉr, D. - 1954: Talajbiologia (Soil Biology), pp. 1-1264. Budapest.

9. Gaffron, H. -1956: Hydrogenase in Algae (Abstract). Plant. Physiol. 31. (Suppl.) XIX: 29.

10. Ge в н A R T, A. -1934: Az abaligeti barlang élövilága, pp. 1-196. Budapest.

11. JAKU Cs, L. - 1952: Az Aggteleki Cseppköbarlang, pp. 1-120. Budapest.

12. — 1953: A Békebarlang felfedezése, pp. 1-145. Budapest.

13. Kessler, E. - 1956: Reduction of nitrite with molecular Hydrogen in algae containing hydrogenase. Arch. Biochem. Biophys. 62: 241-242.

14. Lefevre, M. - 1958: De l'influence des matières organiques sur la nature et l'abondance du plancton. Ann. Station centr. d'Hydrobiol. Appliquée. \%: 251-267.

15. Kou, E. - 1957: Algologiai vizsgálatok a Sátorhegyseg jegesbarlangjában (Algological investigations in the ice cave of the Sátor-Mountain). Bot. Közl. 4\%. (1-2): 43-51.

16. MagdeBuRg, P. - 1929-1932: Organogene Kalkonkretionen in Höhlen. Sitzungber. Nat. Ges., Leipzig 59: 1-26.

17. Morton, F. - 1925: Höhlenpflanzen. Speleologische Monogr. Hrsg. Kyrle 5: 1-16.

18. Palik, P. - 1938: Beiträge zur Kenntnis der lithophyten Algenvegetation des Bükk-Gebirges. Index Horti Bot. Univ. Budapestiensis. 3: 143150 .

19. - 1960: A new blue-green alga from the cave of Baradla, near Aggtelek. Ann. Univ. Sci. Budapestiensis Rol. Eötvös nom. Sect. Biol. 3: 275-285. Budapest.

20. - 1960: Study of the Algae Flora of Caves. Hidr. Közl. 5: 417-422. Budapest.

21. Schwimmer, M. and Sahwimmer, D. -1955 : The role of algae and plankton..., pp. 1-86. New-York, London.

22. Suba, É. - 1957: Die Algen der Pálvölgyer Höhle in Ungarn. Verh. Zool. Bot. Ges., Wien. 97: 97-110. 


\section{ERKLÄRUNG DER TAFEL 9 (1)}

Algenarten der ungarischen Höhlen.

Fig. 1: Gloecapsa punctata Näg. 3000: 1.

Fig. 2: Gloecapsa mellea Kütz. 3000: 1.

Fig. 3: Synechococcus elongatus. Näg. 2000: 1.

Fig. 4 a-f: Baradlaia speluncaecola Palik. - a. Lagerteil. Gerade, steife Fäden mit mehr oder weniger Calzium in den Scheiden. 1000: 1. - b. Die Seitenzweige als Ausschwellungen bemerkbar. 1000:1. - c. Fadenteil mit einem aufrechten Seitenzweig, letzterer etwas gewellt, noch nicht steif. 1000: 1. - d. Teil eines kurzen, verzweigten Fadens, mit Calzium gesättigt. 1000:1. - e. Kolbenartiger Fadenteil mit ungleichmäßiger Kalkablagerung, das obere Ende viel dicker. 2000: 1. - f. Teil eines verzweigten Fadens in beträchtlichem Maße mit Kalk überkrustet. 1000: 1 .

Fig. 5: Oscillatoria tenuis Ag. 1000: 1.

Fig. 6a, b: Oscillatoria animalis Ag. 1000: 1.

Fig. 7 : Symploca muralis Kütz. 1500: 1 .

Fig. 8a, b: Chantransia chalybea (Lyngb.) Fries. 500:1. 


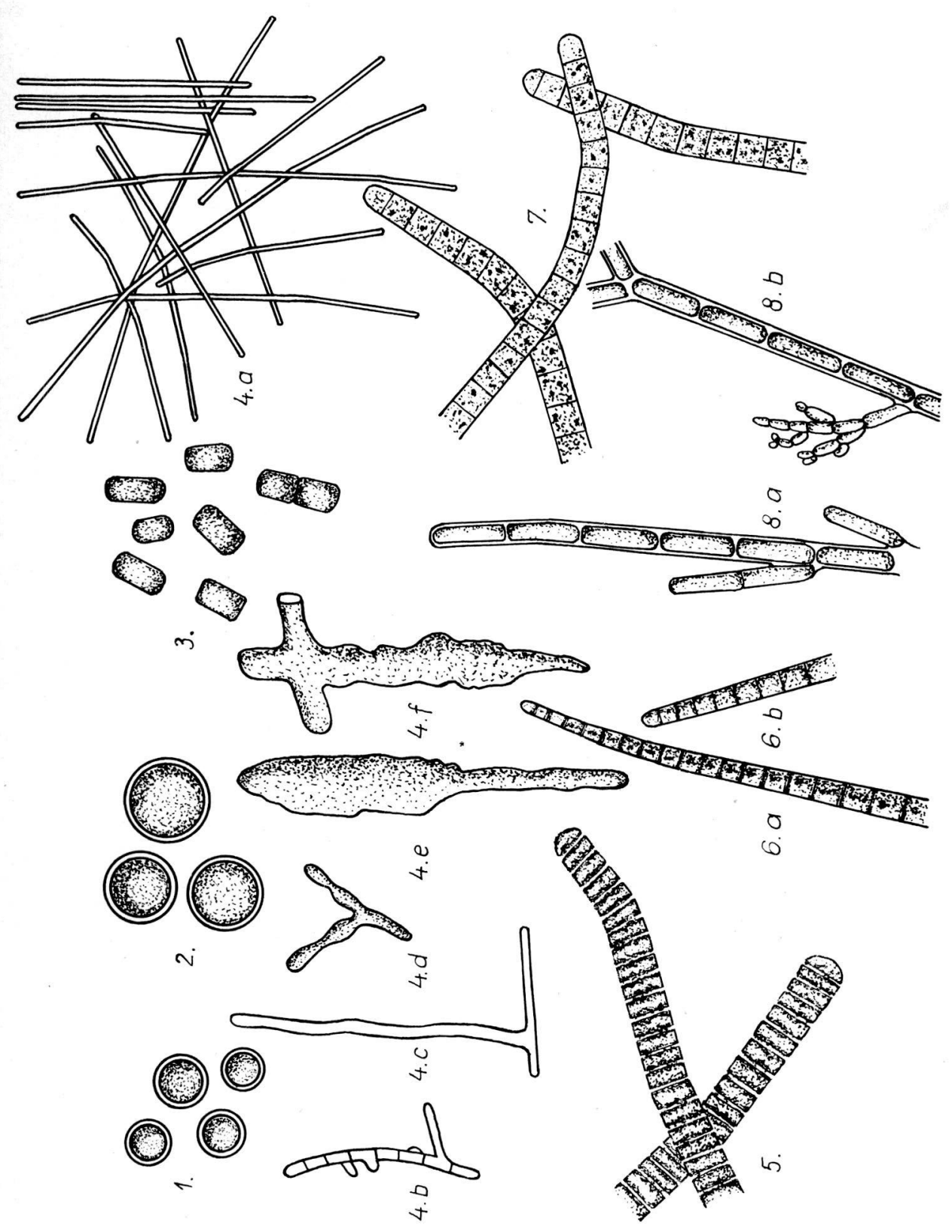

